In der Rubrik „Literatur kompakt" werden die wichtigsten Originalarbeiten aus der internationalen Fachliteratur referiert.

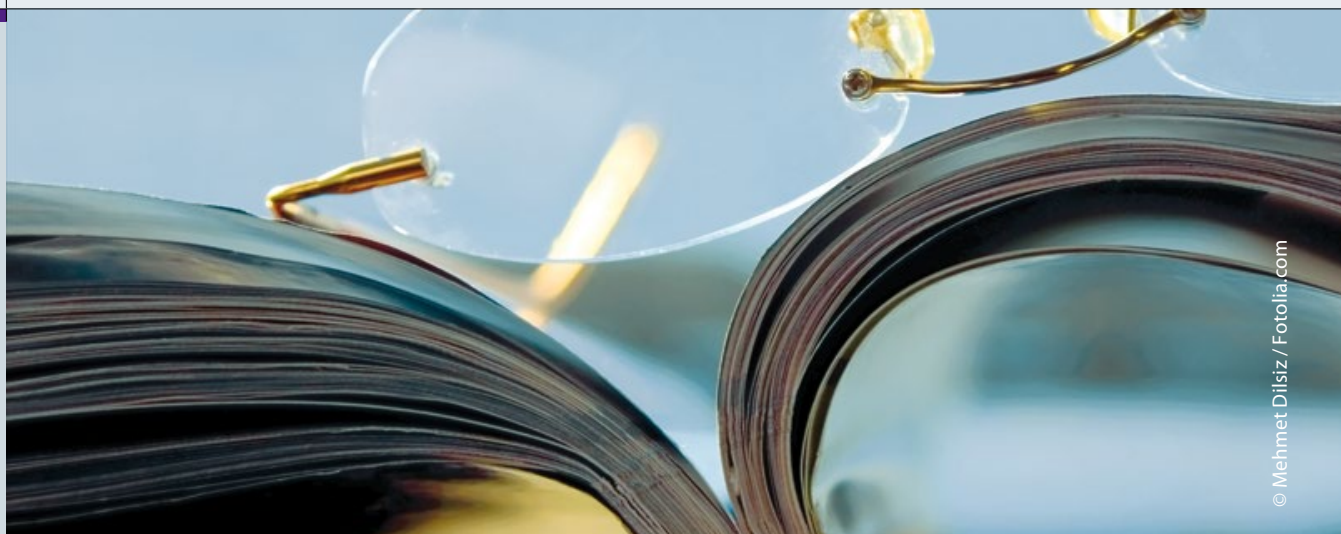

\section{Unfruchtbarkeit: Große Studie bestätigt Chlamydieneffekt}

\author{
Eine dänische Studie bestätigt den negativen Einfluss einer Chlamydien- \\ infektion auf die Fruchtbarkeit.
}

B isherige Studien zum Unfruchtbarkeitsrisiko nach einer Infektion mit Chlamydia trachomatis waren für eine klare Aussage zu klein. Zudem wurden bislang in keiner Untersuchung die drei Komplikationen „pelvic inflammatory disease" (PID), ektope Schwangerschaft und Tubenfaktorinfertilität gemeinsam berücksichtigt. Britische und dänische Infektiologen haben nun Daten der Danish Chlamydia Study mit Befunden aller Frauen mit einem Chlamydientest analysiert, die Bewohnerinnen von Dänemark inklusive Grönland waren. Die Tests wurden zwischen 1995 und 2011 gemacht, seit 1994 bestand in Dänemark
Meldepflicht. Endpunkt der Studie war die Therapie der Frauen in der Klinik aufgrund der drei Komplikationen.

Fast 517.000 Frauen wurden durchschnittlich knapp acht Jahre nachverfolgt. Bei $20 \%$ (103.344 Frauen) war der Chlamydientest positiv, bei 35\% (182.879 Frauen) negativ, und $45 \%$ (230.495 Frauen) waren nie getestet worden. $\mathrm{Zu} \mathrm{Be}$ ginn der Studie waren die Frauen durchschnittlich 22,4 Jahre alt.

Die kumulative Inzidenz für eine PID lag in der Gruppe der positiv getesteten Frauen bei $3,1 \%$, bei negativem Testergebnis bei $2,5 \%$ und bei den nie getesteten Frauen bei 0,6\%. Ähnliche Ergebnis- se ermittelten die Wissenschaftler im Zusammenhang mit der ektopen Schwangerschaft $(2,2 \%, 2,0 \%$ bzw. $0,6 \%$ ). Deutlich niedriger lagen die Raten einer Tubenfaktorinfertilität $(0,6 \%$, $0,5 \%$ bzw. 0,1\%). Bei den Berechnungen der Wahrscheinlichkeiten, dass eine der drei Komplikationen auftritt, berücksichtigten die Wissenschaftler das Alter der Frauen und das Jahr, in dem erstmals Befunde der Teilnehmerinnen für die Studie verwendet worden waren. Im Vergleich zu Studienteilnehmerinnen mit ausschließlich negativen Testergebnissen lag die adjustierte Hazard Ratio (AHR) für eine PID bei Frauen mit einem oder mehreren positiven Testergebnissen bei 1,50. Die Wahrscheinlichkeit für eine PID war somit um $50 \%$ erhöht. Die AHR für eine ektope Schwangerschaft errechneten die Wissenschaftler mit 1,31 und für eine Tubenfaktorinfertilität mit 1,37. Die Berechnungen ergaben auch, dass mehrfache Infektionen mit Chlamydien die Wahrscheinlichkeit für eine PID um weitere $20 \%$ erhöhten (AHR: 1,20). Ein Zusammenhang zwischen Infektionshäufigkeit und Erkrankung wurde dagegen bei ektoper Schwangerschaft oder Tubenfaktorinfertilität nicht beobachtet.

Aufgrund ihrer Studienergebnisse weisen die Autoren darauf hin, wie wichtig es ist, nicht nur Erstinfektionen, sondern auch Nachfolgeinfektionen mit Chlamydia trachomatis zu verhindern, um die Fruchtbarkeit zu erhalten.

Peter Leiner

Davies B et al. Risk of reproductive complications following chlamydia testing: a populationbased retrospective cohort study in Denmark.

The Lancet Infectious Diseases 2016; online 8. Juni. doi: 10.1016/S1473-3099(16)30092-5 